\title{
What is the role of locoregional anesthesia in breast surgery? A systematic literature review focused on pain intensity, opioid consumption, adverse events, and patient satisfaction
}

Pasquale Sansone ${ }^{1 *}$ D, Luca Gregorio Giaccari ${ }^{1}$, Mario Faenza², Pasquale Di Costanzo², Sara Izzo², Caterina Aurilio ${ }^{1}$, Francesco Coppolino ${ }^{1}$, Maria Beatrice Passavanti ${ }^{1}$, Vincenzo Pota ${ }^{1}$ and Maria Caterina Pace ${ }^{1}$

\begin{abstract}
Background: Breast surgery in the United States is common. Pain affects up to 50\% of women undergoing breast surgery and can interfere with postoperative outcomes. General anesthesia is the conventional, most frequently used anaesthetic technique. Various locoregional anesthetic techniques are also used for breast surgeries. A systematic review of the use of locoregional anesthesia for postoperative pain in breast surgery is needed to clarify its role in pain management.

Objectives: To systematically review literature to establish the efficacy and the safety of locoregional anesthesia used in the treatment of pain after breast surgery.

Methods: Embase, MEDLINE, Google Scholar and Cochrane Central Trials Register were systematically searched in Mars 2020 for studies examining locoregional anesthesia for management of pain in adults after breast surgery. The methodological quality of the studies and their results were appraised using the Consensus-based Standards for the Selection of Health Measurement Instruments (COSMIN) checklist and specific measurement properties criteria, respectively.

Results: Nineteen studies evaluating locoregional anesthesia were included: 1058 patients underwent lumpectomy/ mastectomy, 142 breast augmentation and 79 breast reduction. Locoregional anesthesia provides effective anesthesia and analgesia in the perioperative setting, however no statistically significant difference emerged if compared to other techniques. For mastectomy only, the use of locoregional techniques reduces pain in the first hour after the end of the surgery if compared to other procedures $(p=0.02)$. Other potentially beneficial effects of locoregional anesthesia include decreased need for opioids, decreased postoperative nausea and vomiting, fewer complications and increased patient satisfaction. All this improves postoperative recovery and shortens hospitalization stay. In none of these cases, locoregional anesthesia was statistically superior to other techniques.

(Continued on next page)
\end{abstract}

\footnotetext{
* Correspondence: pasquale.sansone@unicampania.it

'Department of Woman, Child and General and Specialized Surgery,

University of Campania "Luigi Vanvitelli", Piazza Luigi Miraglia, 2, Naples, Italy

Full list of author information is available at the end of the article
}

(c) The Author(s). 2020 Open Access This article is licensed under a Creative Commons Attribution 4.0 International License, which permits use, sharing, adaptation, distribution and reproduction in any medium or format, as long as you give appropriate credit to the original author(s) and the source, provide a link to the Creative Commons licence, and indicate if changes were made. The images or other third party material in this article are included in the article's Creative Commons. licence, unless indicated otherwise in a credit line to the material. If material is not included in the article's Creative Commons licence and your intended use is not permitted by statutory regulation or exceeds the permitted use, you will need to obtain permission directly from the copyright holder. To view a copy of this licence, visit http://creativecommons.org/licenses/by/4.0/. The Creative Commons Public Domain Dedication waiver (http://creativecommons.org/publicdomain/zero/1.0/) applies to the data made available in this article, unless otherwise stated in a credit line to the data. 
(Continued from previous page)

Conclusion: The results of our review showed no differences between locoregional anesthesia and other techniques in the management of breast surgery. Locoregional techniques are superior in reducing pain in the first hour after mastectomy.

Keywords: Breast surgery, Mastectomy, Locoregional anesthesia, Pain intensity, Opioid consumption, Adverse events, Patient satisfaction

\section{Background Rationale}

Breast surgery in the United States is common. In 2020, an estimated 276,480 new cases of invasive breast cancer will be diagnosed among women and approximately $80 \%$ of patients will have surgery to remove their primary tumour [1]. In addition, an increasing number of women are turning to plastic surgeons for interventions of cosmetic: among these breast augmentation is the first procedure performed in the United States [2].

Pain affects up to $50 \%$ of women undergoing breast surgery and can interfere with postoperative outcomes. Breast pain is one of the factors determining patient distress, long hospital stay, and an increase in post-surgical admissions to the hospital [3].

Nociceptive/inflammatory pain is caused by tissue damage, whereas neuropathic pain is the consequence of a central and peripheral nerve damage [4], especially the intercostal nerves from T2 to T6. Neuropathic pain typically begins immediately after breast surgery and can be persistent, sometimes even for months [3].

The pain control is the main objective of anesthesia in breast surgery. The correct management of acute postoperative pain is essential to improve patient outcome and satisfaction.

Various anesthetic agents, devices, and strategies are currently available. For a long time, intravenous analgesia has been the main technique for postoperative pain relief. Over the years, the growing number of surgical procedures for breast cancer and cosmetic treatment has however stimulated the development of new anesthetic techniques with improved pain reduction and safety, and fewer complications.

The international guidelines recommended the use of a multimodal analgesia $[5,6]$. Regional anesthesia techniques are effective as a component of multimodal analgesia for management of postoperative pain associated with a number of surgical procedures. These techniques can be administered as a single shot or a continuous catheter, both prior to surgical incision or after surgery $[5,6]$. Local anesthetics infiltration also shows benefit for the surgical procedure. Wound infiltration can be performed either as a single injection of local anesthetic typically at the conclusion of surgery or as a continuous infusion of local anesthetic through a catheter at the incision site prior to skin closure $[5,6]$. Finally, the international guidelines suggest the use of intravenous (IV) lidocaine, especially in patients underwent open or laparoscopic abdominal surgical procedures $[5,6]$. Perioperative lidocaine infusion may be considered for patients undergoing mastectomy [7].

\section{Objectives}

We undertook this systematic review to identify the potential clinical role of locoregional anesthesia for breast surgery. We aimed to establish the efficacy and the safety of locoregional anesthesia in the pain management after breast surgery. Postoperative pain severity and opioid consumption during the first $48 \mathrm{~h}$ were designated as coprimary outcomes. For secondary objectives, we aimed to examine the effects on the immediate quality of recovery, in relation to adverse effects and patient satisfaction.

\section{Methods}

\section{Protocol and registration}

We performed a systematic review based on Preferred Reporting Items for Systematic Reviews and Meta-Analyses (PRISMA) statement [8]. Randomized controlled trials that compared the effects of locoregional anesthesia to systemic analgesia alone in patients undergoing breast surgery were sought. Studies were evaluated using a pre-designed protocol. The protocol was not published, and the review was not registered with the International prospective register of systematic reviews (PROSPERO).

\section{Eligibility criteria}

The population, intervention, comparison, and outcome (PICO) criteria were applied to the research question (see Table 1). Patients older than 18 years undergoing breast surgery were considered as the population (P); the intervention (I) was postoperative analgesia with locoregional anesthesia techniques; the comparison (C) concept was standard pain treatment provided in each study; pain intensity, opioid consumption, adverse events (AEs), and patient satisfaction were considered the outcomes $(\mathrm{O})$ for this systematic review. We included randomized controlled trials (RCTs) published from 2010 to the present. No language restrictions were placed on study inclusion.

\section{Literature search}

We identified the articles by searching electronic databases (Embase, MEDLINE, Google Scholar and Cochrane 
Table 1 PICO criteria for including studies

\begin{tabular}{ll}
\hline Population & Patients of at least 18 years undergoing breast surgery. \\
Intervetion & Postoperative analgesia with locoregional anesthesia techniques. \\
Comparator & Standard pain treatment. \\
Outcomes & Pain Intensity, Opioid Consumption, Adverse Events, Patient Satisfaction. \\
Study TYPE & Randomized Controlled Trial. \\
Time & From 2010 to present. \\
\hline
\end{tabular}

Central Trials Register). We included other relevant studies from the reference lists of systematic reviews and meta-analyses. These databases were initially searched from Mars 2020.

The search strategy was developed using medical subject headings and keywords relating to the central research question of this paper. Namely, the search terms included in the search strategy covered the following key domains: "breast augmentation", "breast reduction", "mastectomy", "mastopexy", "local anesthetic agent", "postoperative pain" and "randomized clinical trial".

We applied no language restrictions in searches.

The studies included in this review evaluated adult patients undergoing breast surgery and receiving any type of locoregional anesthesia to treat postoperative pain.

\section{Primary outcomes}

Pain scores and opioid consumption in the first $48 \mathrm{~h}$ postoperatively were the primary outcomes of interest. Pain intensity was assessed via a Numeric Rating Scale (NRS) at 1, 6, 12, 24 and $48 \mathrm{~h}$ after surgery. Pain intensity data assessed by means other than a zero to 10 NRS were converted to this scale. The other primary outcome was the average per patient opioid consumption in the Post-Anesthesia Care Unit (PACU) and in the $48 \mathrm{~h}$ after surgery. Opioid consumption was converted to morphine sulfate equivalents [9].

\section{Secondary outcomes}

We extracted data on the following secondary outcomes:

1. Adverse Events (AEs) were recorded. Evaluated safety outcomes included postoperative opioid related side-effects (postoperative nausea and vomiting, sedation/respiratory depression, pruritus, hypotension, urinary retention, or constipation), and complications related to the nerve block (pneumothorax, block failure, or local anesthetic systemic toxicity). Complications during wound healing were also analysed.

2. Patient Satisfaction. All measures of patient satisfaction were reported as "satisfied" and "not satisfied".

\section{Selection of studies}

Two independent reviewers (P.S. and L.G.G.) initially assessed the results from the literature search based on title and abstract. The full-text citations of potentially eligible articles were subsequently retrieved and reviewed again by the same two independent reviewers. In case of disagreement between the two reviewers on eligibility, a discussion was initiated. If consensus could not be reached after discussion, a third reviewer (M.C.P.) evaluated the study and made the final decision.

The methodological quality of the included studies was evaluated and rated using the COSMIN checklist, based on a 4-point rating scale.

\section{Data extraction and management}

A standardized data extraction form was used. Data collected included information relating to:

- Age, weight, height and body mass index (BMI) of participants;

- Number of participants enrolled and completing the study;

- Type of operation;

- Regional anesthesia technique (local anesthetic and dose);

- Pain intensity for all-time points at which it was measured;

- Opioid consumption;

- Patient satisfaction;

- Severity or incidence of adverse events.

\section{Statistical analyses}

For continuous outcomes, we extracted the mean and standard deviation (SD). In situations where these are not reported, the median and interquartile range were used to approximate these values. Similarly, in situations where the mean and 95\% CI are reported, statistical conversions were used to estimate the mean and SD.

For dichotomous outcomes (side effects, complications), data were converted to overall incidence numbers.

We designated a $p$ value $<0.05$ as a threshold of statistical significance for the primary and secondary outcomes. All tests were two-tailed. 


\section{Results}

Our search strategy identified 40 citations. Searching additional sources yielded an additional 9 potentially eligible citations. Of these, 30 were excluded based on title and abstract screening, because of duplicated papers $(n=5)$, missing outcomes $(n=7)$, or the lack of a standardized pain treatment in the control group $(n=18)$. Thus a total of 19 randomized controlled trials were included in this systematic review. The flow diagram (see Fig. 1) shows the results from the literature search and the study selection process.

The characteristics of included studies and outcomes assessed in this review are presented in Table 2a, b and c.

The surgical procedures performed in the reviewed trials included lumpectomy or mastectomy in 14 of 19 trials [10-23], with additional Sentinel Lymph Node Biopsy (SLNB) or Axillary Lymph Node Dissection (ALND) [10, 12, 14-18, 20, 21, 23]. Three trials included patients undergoing breast augmentation [24-26], and two trials also included patients undergoing breast reduction $[27,28]$.
The 19 randomized controlled trials involved a total of 1307 patients, of which 749 received nerve blocks, 330 received local infiltration, 115 received IV lidocaine, 73 received infusion of local anesthetic via pump, and 40 received tumescent anesthesia.

According to the COSMIN checklist, all studies included in this review showed an excellent-to-good quality. The majority of clinical trials had a low risk of bias.

\section{Lumpectomy/mastectomy}

In the included studies 1086 patients underwent lumpectomy/mastectomy. There were no demographic differences between the two groups as shown in Table 3.

The largest studies involved 140 patients (Mohamed et al., [17]; Versyck et al., [20]), whereas the smallest consisted of 44 patients (Couceiro et al., [11]). All analysed studies were conducted in inpatient settings.

Patients underwent mastectomy, while lumpectomy was performed in 4 studies (Campbell et al.,[10]; Cros et al., [12]; Gürkan et al.,[14]; Versyck et al., [20]). Lanier et al [16] and Wang et al [21] reported immediate tissue

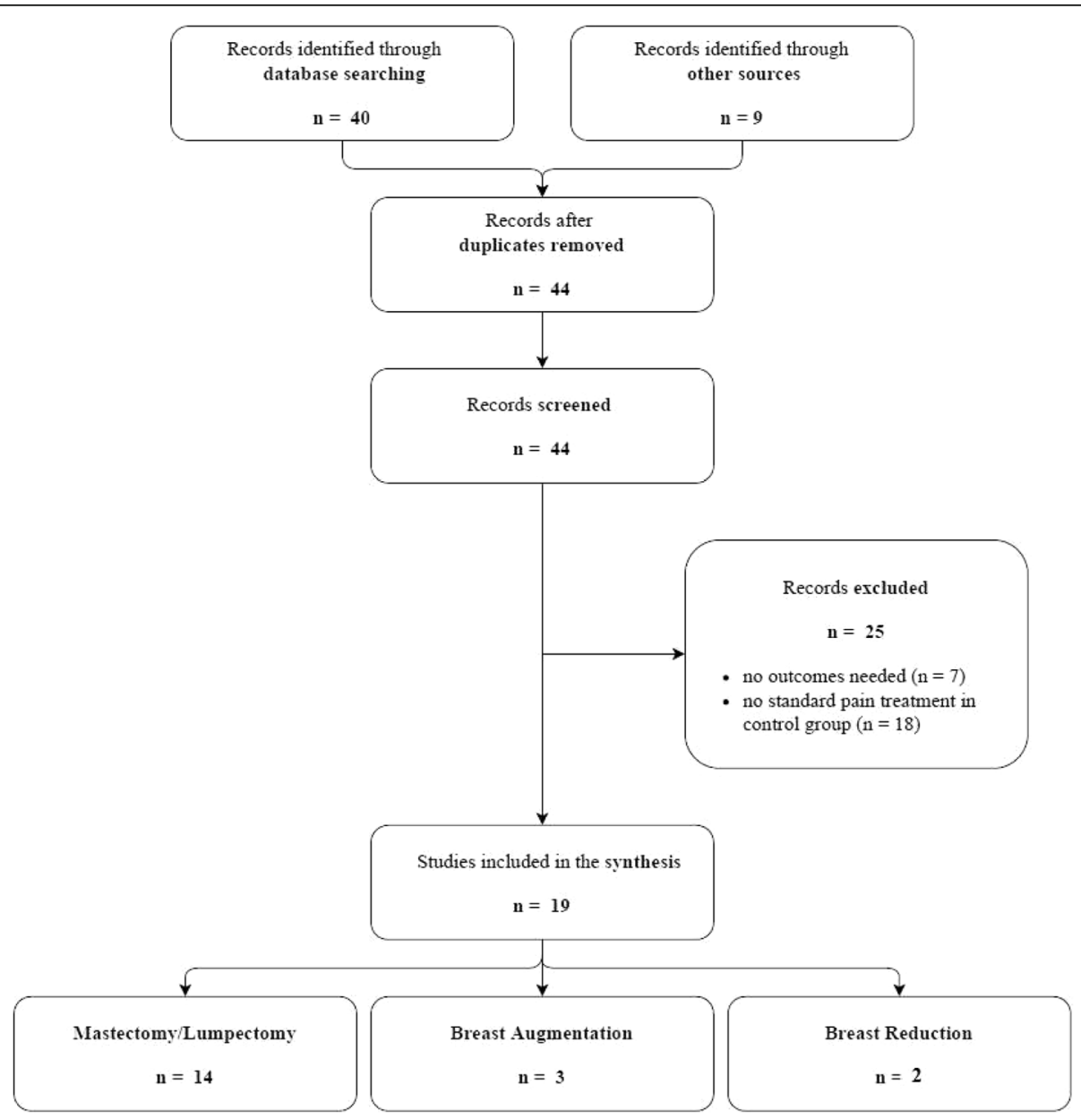

Fig. 1 Flow diagram study selection process 
Table 2 Studies characteristics

\begin{tabular}{|c|c|c|c|c|c|c|}
\hline Author, year & Study & Sites & No. & Type of surgery & Anesthesia & Intervention \\
\hline \multicolumn{7}{|c|}{ A. LUMPECTOMY/MASTECTOMY } \\
\hline Campbell et al.,[10] 2014 & SB-RCT & New Zeland, 1 & 79 & Lumpectomy/Mastectomy \pm ALND & $?$ & Local Infiltration \\
\hline Couceiro et al.,[11] 2014 & DB-RCT & Brazil, 1 & 44 & Mastectomy & GA & IV lidocaine \\
\hline Cros et al.,[12] 2018 & DB-RCT & $\begin{array}{l}\text { France, } 1 \\
\text { Canada, } 1\end{array}$ & 127 & Lumpectomy/Mastectomy \pm SLNB/ALND & GA & Pecs 1 \\
\hline Ferreira Laso et al.,[13] 2014 & DB-RCT & Spain, 1 & 73 & Mastectomy & GA & Infusion pump \\
\hline Gürkan et al.,[14] 2018 & SB-RCT & Turkey,? & 50 & Lumpectomy/Mastectomy \pm SLNB/ALND & GA & ESP \\
\hline Ilfeld et al.,[15] 2014 & DB-RCT & US,? & 60 & Mastectomy \pm ALND & Sedation & PVB \\
\hline Lanier et al .[16]2018 & DB-RCT & US,? & 45 & Mastectomy + reconstruction \pm SLNB/ALND & $?$ & $\begin{array}{l}\text { Intercostal + pectoral } \\
\text { nerve blocks }\end{array}$ \\
\hline Mohamed et al.,[17] 2013 & DB-RCT & Egypt,? & 140 & Mastectomy \pm ALND & GA & Local infiltration \\
\hline Neethu et al.,[18] 2018 & $\mathrm{RCT}$ & India, 1 & 60 & Mastectomy \pm SLNB/ALND & GA & Pecs I - II \\
\hline Terkawi et al.,[19]2014 & DB-RCT & US,? & 71 & Mastectomy & GA & IV lidocaine \\
\hline Versyck et al.,[20] 2017 & DB-RCT & Belgium, 1 & 140 & Mastectomy/tumorectomy \pm SLNB/ALND & GA & Pecs $\|$ \\
\hline Wang et al.,[21] 2018 & SB-RCT & China,? & 64 & Mastectomy + reconstruction \pm ALND & GA & Pecs ॥ \\
\hline Wang et al.,[22] 2019 & $\mathrm{RCT}$ & China,? & 61 & Mastectomy & GA & Pecs I+SPB \\
\hline Yao et al.,[23] 2019 & DB-RCT & China, 1 & 72 & Mastectomy \pm ALND & GA & SPB \\
\hline \multicolumn{7}{|l|}{ B. BREAST AUGMENTATION } \\
\hline Gardiner et al.,[24] 2012 & SB-RCT & Australia,? & 40 & BA & Sedation & PVB \\
\hline Picard et al.,[25] 2017 & SB-RCT & France,? & 72 & BA & $?$ & Local infiltration \\
\hline Schuitemaker et al.,[26] 2019 & DB-RCT & Spain,? & 30 & BA & GA & Pecs II +SPB \\
\hline \multicolumn{7}{|l|}{ C. BREAST REDUCTION } \\
\hline Christie et al., [27] 2017 & DB-RCT & US, 1 & 40 & $B R$ & GA & Tumescent Anaesthesia \\
\hline Valente et al.,[28] 2014 & DB-RCT & Brazil,? & 39 & $B R$ & GA & Local infiltration \\
\hline
\end{tabular}

SB-RCT Single-Blind Randomized Controlled Trial, DB-RCT Double-Blind Randomized Controlled Trial, SLNB Sentinel Lymph Node Biopsy, ALND Axillary Lymph Node Dissection, GA General Anesthesia, Pecs Pectoralis nerve block, ESP Erector Spinae Plane block, PVB ParaVertebral Block, SPB Serratus Plane Block, BA Breast

Augmentation, $B R$ Breast Reduction;?, not reported

expander or implant based breast reconstruction. Surgical treatment for breast cancer was associated with a sentinel lymph node biopsy (SLNB) or a axillary lymph node dissection (ALND) in 10 papers (Campbell et al.,[10]; Cros et al.,[12]; Gürkan et al.,[14]; Ilfeld et al., [15]; Lanier et al., [16]; Mohamed et al.,[17]; Neethu et al., 2018; Versyck et al. [20]; Wang et al., 2018; Yao et al, 2019).

Regional anesthetic techniques were performed ten times: the most common procedure was pectoral nerve (Pecs) block type I and II (5 studies: Cros et al.,[12]; Neethu et al., 2018; Versyck et al. 2017; Wang et al., 2018; Wang et al., 2019), followed by serratus plane block (SPB) (2 studies: Wang et al., 2019; Yao et al., 2019), erector spinae plane (ESP) block (Gürkan et al., [14]), and paravertebral block (PVB) (Ilfeld et al.,[15]). In Lanier et al 2018, intraoperative nerve blocks of intercostal and pectoral nerves were performed under direct visualization by the attending plastic surgeon at the completion of the mastectomy.

Local infiltration was reported in 3 studies. In Campbell et al 2014 [10], patients received $20 \mathrm{~mL}$ of bupivacaine $0.25 \%$ with or without adrenaline to be infiltrated into the breast wound and a further $20 \mathrm{~mL}$ of bupivacianee $0.25 \%$ with adrenaline to be infiltrated into the axilla wound when applicable. In Mohamed et al 2013, $5 \mathrm{ml}$ of bupivacaine $0.5 \%$ with or without clonidine were diluted with saline $0.9 \%$ to $15 \mathrm{~mL}$ volume and irrigated into the surgical field before skin closure. An infusion pump of levobupivacaine $0.50 \%$ for approximately $48 \mathrm{~h}$ was evaluated in Ferreira Laso et al 2014.

Couceiro et al [11] and Terkawi et al 2014 investigated i.v. lidocaine infusion. In the first paper, a bolus dose of lidocaine was not administered and, after incision, a lidocaine infusion at $3 \mathrm{mg} / \mathrm{kg}$ was started. In the other study, lidocaine was administered as a bolus to all patients before anesthetic induction, at a dose of up to 1.5 $\mathrm{mg} / \mathrm{kg}$, followed by a lidocaine infusion at $2 \mathrm{mg} / \mathrm{kg} / \mathrm{h}$ until $2 \mathrm{~h}$ after arrival in PACU.

Almost all studies were conducted under general anesthesia, except for Ilfeld et al 2014 conducted under sedation. General anesthesia was induced and then maintained with opioids, such as alfentanil (Ferreira 
Table 3 Personal and clinical characteristics of patients undergoing lumpectomy/mastectomy

\begin{tabular}{|c|c|c|c|c|c|c|c|c|c|c|}
\hline & \multicolumn{2}{|c|}{ Patients ( $n=)$} & \multicolumn{2}{|l|}{ Age (years) } & \multicolumn{2}{|l|}{ Weight (kg) } & \multicolumn{2}{|l|}{ Height $(\mathrm{cm})$} & \multicolumn{2}{|l|}{ BMI $\left(\mathrm{kg} / \mathrm{m}^{2}\right)$} \\
\hline & Group I & Group C & Group I & Group C & Group I & Group C & Group I & Group C & Group I & Group C \\
\hline $\begin{array}{l}\text { Campbell et al., } \\
2014 \text { [10] }\end{array}$ & 45 & 34 & 59.4 & 61.7 & 80.7 & 73.8 & 163.3 & 162.3 & 30.2 & 28.1 \\
\hline $\begin{array}{l}\text { Couceiro et al., } \\
2014[11]\end{array}$ & 22 & 22 & 47.0 & 52.4 & - & - & - & - & 28.1 & 28.2 \\
\hline $\begin{array}{l}\text { Cros et al., } \\
2018 \text { [12] }\end{array}$ & 62 & 66 & 60.5 & 62.0 & 63.6 & 65.0 & 160.0 & 160.0 & 24.8 & 25.6 \\
\hline $\begin{array}{l}\text { Ferreira Laso et al., } \\
2014 \text { [13] }\end{array}$ & 34 & 39 & 54.8 & 57.7 & 67.2 & 66.7 & - & - & - & - \\
\hline $\begin{array}{l}\text { Gürkan et al., } \\
2018[14]\end{array}$ & 25 & 25 & 49.5 & 49.8 & 72.4 & 73.1 & 161.0 & 161.0 & 27.8 & 28.2 \\
\hline $\begin{array}{l}\text { Ilfeld et al., } \\
2014 \text { [15] }\end{array}$ & 30 & 30 & 48.0 & 49.0 & 62.0 & 61.0 & 165.0 & 166.0 & 23.0 & 24.0 \\
\hline $\begin{array}{l}\text { Lanier et al., } \\
2018[16]\end{array}$ & 23 & 22 & 48.0 & 50.0 & 67.0 & 70.0 & 160.0 & 170.0 & 26.0 & 26.0 \\
\hline $\begin{array}{l}\text { Mohamed et al., } \\
2013 \text { [17] }\end{array}$ & 105 & 35 & 39.9 & 38.9 & 70.2 & 69.8 & 160.6 & 158.5 & 27.4 & 27.9 \\
\hline $\begin{array}{l}\text { Neethu et al., } \\
2018 \text { [18] }\end{array}$ & 30 & 30 & 50.5 & 45.6 & - & - & - & - & - & - \\
\hline $\begin{array}{l}\text { Terkawi et al., } \\
2014[19]\end{array}$ & 34 & 37 & 53.0 & 54.0 & - & - & - & - & 26.2 & 28.2 \\
\hline $\begin{array}{l}\text { Versyck et al., } \\
2017[20]\end{array}$ & 45 & 40 & 59.6 & 58.8 & 67.3 & 67.0 & 165.0 & 165.0 & 24.7 & 24.6 \\
\hline $\begin{array}{l}\text { Wang et al., } \\
2018 \text { [21] }\end{array}$ & 30 & 30 & 46.8 & 47.4 & - & - & - & - & 25.4 & 24.8 \\
\hline $\begin{array}{l}\text { Wang et al., } \\
2019 \text { [22] }\end{array}$ & 32 & 29 & 51.3 & 55.3 & 58.7 & 57.6 & 162.5 & 161.0 & 22.3 & 22.2 \\
\hline $\begin{array}{l}\text { Yao et al., } \\
2019[23]\end{array}$ & 34 & 34 & 46.5 & 47.7 & 57.2 & 56.2 & 160.9 & 160.8 & 22.3 & 21.9 \\
\hline TOTAL & 585 & 473 & $51.05 \pm 5.6$ & $52.16 \pm 6.3$ & $66.65 \pm 6.5$ & $66.03 \pm 5.8$ & $162.04 \pm 1.8$ & $162.74 \pm 3.3$ & $25.71 \pm 2.3$ & $25.83 \pm 2.2$ \\
\hline
\end{tabular}

Laso et al., 2014), fentanyl (Couceiro et al., [11]; Gürkan et al., 2018; Ilfeld et al., 2014; Mohamed et al., 2013; Neethu et al., 2018; Terkawi et al., 2014; Wang et al., 2018), remifentanil (Wang et al., 2019) and sufentanil (Versyck et al., 2017; Wang et al., 2019; Yao et al., 2019). For the postoperative pain management, four studies (Couceiro et al., [11]; Cros et al.,[12]; Ferreira Laso et al., 2014; Ilfeld et al., 2014) provided infiltration of the chest wall ipsilateral to the mastectomy with local anesthetic; acetaminophen and other NSAIDs were systematically administered. Two studies did not report the anesthesia protocol (Campbell et al., 2014 [10]; Lanier et al., 2018).

\section{Pain intensity}

Different investigators recorded this outcome on different scales and at different intervals. We normalized all NRS to a zero to 10 range (see Table 4). The majority of authors reported pain intensity at 1, 6, 12, 24 and $48 \mathrm{~h}$ after surgery.

As shown in Fig. 2, the average NRS score was $1.83 \pm$ 0.81 at $1 \mathrm{~h}, 2.02 \pm 1.03$ at $6 \mathrm{~h}, 1.92 \pm 1.57$ at $12 \mathrm{~h}, 1.74 \pm$ 1.29 at $24 \mathrm{~h}$ and $1.51 \pm 1.03$ at $48 \mathrm{~h}$ in the intervention group; in the placebo group NRS scores were recorded of $3.36 \pm 1.65$ at $1 \mathrm{~h}, 3.10 \pm 1.29$ at $6 \mathrm{~h}, 2.35 \pm 1.14$ at 12 h, $2.37 \pm 1.27$ at $24 \mathrm{~h}$ and $2.77 \pm 1.12$ at $48 \mathrm{~h}$.

One study, Couceiro et al [11], did not report NRS at any interval: only $2(9.09 \%)$ vs $3(13.6 \%)$ patients in the lidocaine and placebo groups, respectively, experienced severe to very severe pain $24 \mathrm{~h}$ after surgery.

\section{Opioid consumption}

The most frequently used opioids were codeine (Campbell et al., 2014 [10]; Couceiro et al., [11]), fentanyl (Neethu et al., 2018), oxycodone (Campbell et al., 2014) [10], piritramide (Versyck et al. 2017), tramadol (Campbell et al., 2014 [10]; Mohamed et al., 2013; Versyck et al. 2017), and sufentanil (Yao et al., 2019). Morphine was used in the other studies.

At discharge from PACU, the overall mean amounts of morphine consumed in the intervention and placebo groups were $3.0 \pm 3.63 \mathrm{mg}$ and $4.87 \pm 5.76 \mathrm{mg}$, respectively, with the difference being not statistically significant $(p=0.51)$.

After $48 \mathrm{~h}$, the overall mean amounts of morphine consumed in the intervention and placebo groups were, 
Table 4 NRS at 1, 6, 12, 24 and $48 \mathrm{~h}$ after lumpectomy/mastectomy

\begin{tabular}{|c|c|c|c|c|c|c|c|c|c|c|}
\hline & \multicolumn{2}{|l|}{ Up to $1 \mathrm{~h}$} & \multicolumn{2}{|l|}{ Up to $6 \mathrm{~h}$} & \multicolumn{2}{|l|}{ Up to $12 \mathrm{~h}$} & \multicolumn{2}{|l|}{ Up to $24 \mathrm{~h}$} & \multicolumn{2}{|l|}{ Up to $48 \mathrm{~h}$} \\
\hline & Group I & Group C & Group I & Group C & Group I & Group C & Group I & Group C & Group I & Group C \\
\hline $\begin{array}{l}\text { Campbell et al., } \\
2014 \text { [10] }\end{array}$ & - & - & 1.75 & 2 & - & - & 2.3 & 1.7 & 1.8 & 1.25 \\
\hline $\begin{array}{l}\text { Couceiro et al., } \\
2014[11]\end{array}$ & - & - & - & - & - & - & - & - & - & - \\
\hline $\begin{array}{l}\text { Cros et al., } \\
2018 \text { [12] }\end{array}$ & 3 & 3 & - & - & - & - & - & - & - & - \\
\hline $\begin{array}{l}\text { Ferreira Laso et al., } \\
2014 \text { [13] }\end{array}$ & 1.6 & 6.7 & - & - & - & - & 0.8 & 4.2 & 0.4 & 3.3 \\
\hline $\begin{array}{l}\text { Gürkan et al., } \\
2018[14]\end{array}$ & 2 & 2 & 2 & 2 & 0 & 1 & 0 & 1 & - & - \\
\hline $\begin{array}{l}\text { Ilfeld et al., } \\
2014 \text { [15] }\end{array}$ & - & - & - & - & - & - & 3.6 & 3.7 & - & - \\
\hline $\begin{array}{l}\text { Lanier et al., } \\
2018[16]\end{array}$ & 3 & 5 & 4 & 5 & 5 & 4 & 4 & 4 & - & - \\
\hline $\begin{array}{l}\text { Mohamed et al., } \\
2013 \text { [17] }\end{array}$ & 2.67 & 3.7 & 2.43 & 3.6 & 2.53 & 3.8 & 2.43 & 3.7 & 2.43 & 3.8 \\
\hline $\begin{array}{l}\text { Neethu et al., } \\
2018 \text { [18] }\end{array}$ & 1.78 & 3.08 & 0.43 & 0.76 & 1.20 & 1.40 & 0.5 & 0.53 & - & - \\
\hline $\begin{array}{l}\text { Terkawi et al., } \\
2014 \text { [19] }\end{array}$ & - & - & 2.94 & 3.88 & - & - & 2.91 & 2.66 & 2.72 & 3.9 \\
\hline $\begin{array}{l}\text { Versyck et al., } \\
2017[20]\end{array}$ & 0.38 & 0.20 & - & - & - & - & - & - & - & - \\
\hline $\begin{array}{l}\text { Wang et al., } \\
2018 \text { [21] }\end{array}$ & 1.65 & 4.3 & 2.45 & 3.9 & 1.8 & 1.9 & 1 & 1 & - & - \\
\hline $\begin{array}{l}\text { Wang et al., } \\
2019 \text { [22] }\end{array}$ & 1 & 3 & 0.8 & 4.4 & 1 & 2 & 0.5 & 1.8 & 0.2 & 1.6 \\
\hline $\begin{array}{l}\text { Yao et al., } \\
2019[23]\end{array}$ & 1.3 & 2.7 & 1.4 & 2.4 & - & - & 1.2 & 1.8 & - & - \\
\hline TOTAL & $1.83 \pm 0.81$ & $3.36 \pm 1.65$ & $2.02 \pm 1.03$ & $3.10 \pm 1.29$ & $1.92 \pm 1.57$ & $2.35 \pm 1.14$ & $1.74 \pm 1.29$ & $2.37 \pm 1.27$ & $1.51 \pm 1.03$ & $2.77 \pm 1.12$ \\
\hline$p$ & $0.02^{*}$ & & 0.08 & & 0.63 & & 0.29 & & 0.13 & \\
\hline
\end{tabular}

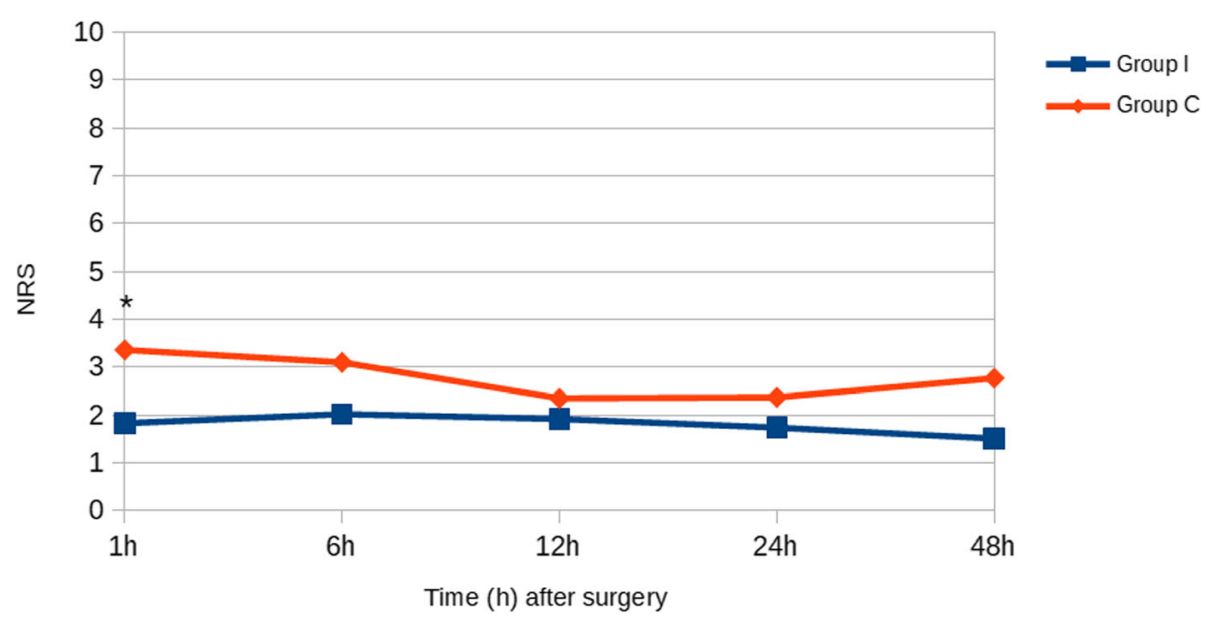

Fig. 2 NRS at 1, 6, 12, 24 and $48 \mathrm{~h}$ after lumpectomy/mastectomy 
respectively, $2.68 \pm 0.88 \mathrm{mg}$ and $4.94 \pm 4.61 \mathrm{mg}(p=0.18)$. Among regional anesthetic techniques, postoperative opioid consumption for the first $48 \mathrm{~h}$ was respectively $2.14 \pm 1.52 \mathrm{mg}$ and $4.84 \pm 4.63 \mathrm{mg}$; no statistically significant difference was observed $(p=0.16)$. The average per patient opioid consumption up to $48 \mathrm{~h}$ after surgery was $1.33 \pm 1.49 \mathrm{mg}$ vs $3.01 \pm 3.05 \mathrm{mg}(p=0.52)$ among patients treated with local infiltration.

Table 5 shows the mean amounts of morphine consumed in the intervention and placebo groups.

In Couceiro et al [11], opioid consumption in the first $24 \mathrm{~h}$ after surgery was similar in the lidocaine and placebo groups.

\section{Adverse events (AEs)}

An adverse event is defined as any undesirable experience associated with the use of a medical product in a patient. A total of $379 \mathrm{AEs}$ were recorded. Three studies (Couceiro et al., [11]; Ilfeld et al., 2014; Versyck et al., 2017) did not report the number of AEs. The most frequently reported AEs were nausea, vomiting and postoperative nausea and vomiting (PONV), pruritus, dizziness, haematoma/bleeding, seroma and bruising (see Table 6 and Fig. 3). Some studies did not specify the timing of adverse events.

\section{Nausea, vomiting and PONV}

Nausea, vomiting and PONV were the most frequent AEs (235 events, 62\% of AEs). 96 vs 139 episodes were respectively recorded in intervention vs placebo group $(p=0.25)$. In 9 studies (Cros et al.,[12]; Ferreira Laso et al., 2014; Gürkan et al., 2018; Lanier et al., 2018; Neethu et al., 2018; Terkawi et al., 2014; Versyck et al. 2017; Wang et al., 2018; Yao et al., 2019) prophylaxis was administered; in the others studies prescription of antiemetic drugs was missed or not reported.

\section{Pruritus}

A total of 23 cases of pruritus was reported in two studies (Ferreira Laso et al., 2014; Wang et al., 2019). No statistically significant difference was observed ( 7 vs $16, p=0.47$ ).

\section{Dizziness}

Fifteen episodes were recorded among patients not receiving treatment; only two patients reported dizziness in the intervention group. No statistically significant difference $(p=0.06)$ was observed in these 3 studies (Ferreira Laso et al., 2014; Wang et al., 2019; Yao et al., 2019).

\section{Haematoma/bleeding}

Three studies (Campbell et al., 2014 [10]; Cros et al.,[12]; Ferreira Laso et al.) reported a total of 21 episodes (10 vs $11, p=0.85)$.

\section{Seroma}

Seroma was found in 10 cases in the intervention group vs 11 cases in the placebo group (Campbell et al., 2014 [10]; Ferreira Laso et al., 2014) with no statistically significant difference $(p=0.91)$.

Table 5 Average per patient opioid consumption in PACU and up to $48 \mathrm{~h}$ after lumpectomy/mastectomy

\begin{tabular}{|c|c|c|c|c|c|c|}
\hline & \multicolumn{3}{|l|}{ PACU } & \multicolumn{3}{|l|}{ Up to $48 \mathrm{~h}$} \\
\hline & Group I & Group C & $p$ & Group I & Group C & $p$ \\
\hline Campbell et al., 2014 [10] & - & - & & 3.42 & $7.33^{\mathrm{a}}$ & \\
\hline Couceiro et al., 2014 [11] & - & - & & - & - & \\
\hline Cros et al., 2018 [12] & 1.5 & 3 & & 1.5 & 3 & \\
\hline Ferreira Laso et al., 2014 [13] & 0 & $0.7^{\mathrm{a}}$ & & 0 & $0.7^{\mathrm{a}}$ & \\
\hline Gürkan et al., 2018 [14] & 1 & 1 & & 5 & $16^{a}$ & \\
\hline Ilfeld et al., 2014 [15] & 1 & $2.4^{\mathrm{a}}$ & & 2.5 & 5.7 & \\
\hline Lanier et al., 2018 [16] & 8 & 17 & & 4 & 5.18 & \\
\hline Mohamed et al., 2013 [17] & - & - & & 0.58 & $1^{a}$ & \\
\hline Neethu et al., 2018 [18] & - & - & & 1.46 & $2.03^{\mathrm{a}}$ & \\
\hline Terkawi et al., 2014 [19] & 9.35 & 9.69 & & 11.02 & 11.61 & \\
\hline Versyck et al., 2017 [20] & 0.18 & $0.33^{\mathrm{a}}$ & & 0.20 & $0.37^{\mathrm{a}}$ & \\
\hline Wang et al., 2018 [21] & - & - & & 1.75 & $5.42^{\mathrm{a}}$ & \\
\hline Wang et al., 2019 [22] & - & - & & - & - & \\
\hline Yao et al., 2019 [23] & - & - & & 0.73 & $1.03^{\mathrm{a}}$ & \\
\hline TOTAL & $3.0 \pm 3.63$ & $4.87 \pm 5.76$ & 0.51 & $2.68 \pm 0.88$ & $4.94 \pm 4.61$ & 0.18 \\
\hline
\end{tabular}

${ }^{a}$ Difference statistically significant 
Table 6 Number of adverse events (AEs) after lumpectomy/ mastectomy

\begin{tabular}{llll}
\hline AEs & $\begin{array}{l}\text { Group I } \\
(\boldsymbol{n}=)\end{array}$ & $\begin{array}{l}\text { Group C } \\
(\boldsymbol{n}=)\end{array}$ & $\boldsymbol{p}$ \\
\hline Nausea/Vomiting/PONV & 96 & 139 & 0.25 \\
Pruritus & 7 & 16 & 0.47 \\
Hypotension & 5 & 2 & 0.25 \\
Hypertension & 0 & 1 & 0.32 \\
Dizzness & 2 & 15 & 0.06 \\
Bradycardia & 2 & 0 & 0.32 \\
Hematoma/Bleeding & 10 & 11 & 0.85 \\
Seroma & 10 & 11 & 0.91 \\
Alteration of healing & 2 & 3 & 0.78 \\
Infection & 3 & 2 & 0.69 \\
DVT & 1 & 1 & 1.00 \\
PTE & 1 & 1 & 1.00 \\
Acute respiratory infection & 1 & 1 & 1.00 \\
Bruising & 23 & 13 & 0.70 \\
TOTAL & $\mathbf{1 6 3}$ & $\mathbf{2 1 6}$ & $\mathbf{0 . 7 4}$ \\
\hline
\end{tabular}

\section{Bruising}

Campbell et al 2014 [10] reported 36 episodes of bruising (20 vs $16, p=0.70)$.

\section{Others}

Haemodynamic changes were rarely reported. Hypotension was reported in 3 studies (Cros et al., [12]; Ferreira Laso et al., 2014; Mohamed et al., 2013) for a total of 7 AEs (5 vs 2, $p=0.25)$. Ferreira Laso et al 2014 reported a case of hypertension. Two episodes of bradycardia were reported from Mohamed et al 2013.

Infection was observed, respectively, 3 vs 2 times in intervention and placebo groups in two studies (Campbell et al., 2014 [10]; Ferreira Laso et al.); no statistically significant difference was noticed $(p=0.69)$. Deep vein thrombosis (DVT), pulmonary thromboembolism (PTE) and acute respiratory infection were equally distributed ( 1 vs $1, p=1.0$ ) in Ferreira Laso et al 2014.

\section{Patient satisfaction}

Patient satisfaction results were presented as different degrees of subjective satisfaction levels. We normalized all of them to "satisfied / not satisfied".

Only 5 studies (Cros et al.,[12]; Ferreira Laso et al., 2014; Lanier et al., 2018; Neethu et al., 2018; Wang et al., 2019;) were available for analysis of satisfaction (see Table 7 and Fig. 4).

Among the intervention group, there were 164 satisfied patients vs 14 not satisfied patients; in the placebo group 149 patients were satisfied and 40 not satisfied. No statistically significant difference was observed between the two groups (satisfied, $p=0.28$; not satisfied, $p=0.14)$.

In Versyck et al 2017, both patient-groups were very satisfied with their management; while in Yao et al 2019, patient satisfaction scores were higher in the SPB group.

\section{Breast augmentation}

One hundred forty-two patients underwent breast augmentation. As shown in Table 8, the groups were similar in age, weight, height and body mass index.

All patients underwent subpectoral bilateral cosmetic breast augmentation.

Regional anesthetic techniques were performed in two studies: PVB in Gardiner et al [24]; association of Pecs type II and PSB in Schuitemaker et al [26]. In the other study, Picard et al [25], a local infiltration was performed.

Patients received general anesthesia in Schuitemaker et al [26], and sedation in Gardiner et al [24]. In both studies patients received fentanyl. Picard et al [25] did not report the anesthesia protocol.

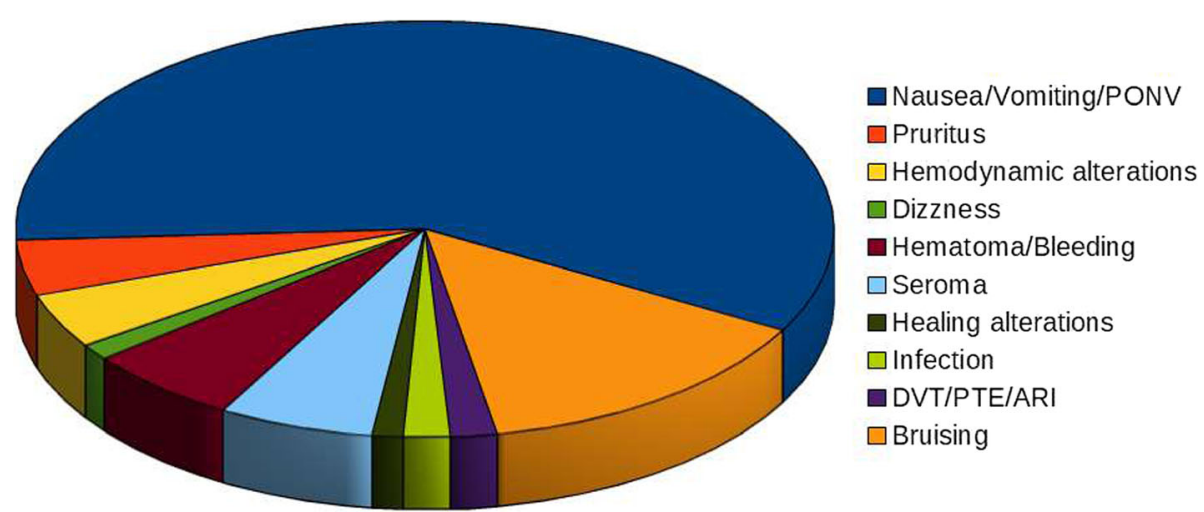

Fig. 3 Distribution of adverse events (AEs) among intervention group (lumpectomy/mastectomy) 
Table 7 Patient satisfaction after lumpectomy/mastectomy

\begin{tabular}{|c|c|c|c|c|}
\hline & Group I & & Group C & \\
\hline & Satisfied & Not satisfied & Satisfied & Not satisfied \\
\hline Campbell et al., 2014 [10] & - & - & - & - \\
\hline Couceiro et al., 201 4[11] & - & - & - & - \\
\hline Cros et al., 2018 [12] & 61 & 1 & 64 & 1 \\
\hline Ferreira Laso et al., 2014 [13] & 32 & 2 & 37 & 2 \\
\hline Gürkan et al., 2018 [14] & - & - & - & - \\
\hline Ilfeld et al., 2014 [15] & - & - & - & - \\
\hline Lanier et al., 2018 [16] & 23 & 0 & 23 & 0 \\
\hline Mohamed et al., 2013 [17] & - & - & - & - \\
\hline Neethu et al., 2018 [18] & 25 & 5 & 10 & 20 \\
\hline Terkawi et al., 2014 [19] & - & - & - & - \\
\hline Versyck et al., $2017^{a}[20]$ & - & - & - & - \\
\hline Wang et al., 2018 [21] & - & - & - & - \\
\hline Wang et al., 2019 [22] & 23 & 6 & 15 & 17 \\
\hline Yao et al., $2019^{b}$ & - & - & - & - \\
\hline TOTAL & 164 & 14 & 149 & 40 \\
\hline
\end{tabular}

a Both patient-groups were very satisfied about their management (9.6 \pm 0.6 and $9.1 \pm 1.8$ on a 10-point scale, $p=0.21)$

${ }^{b}$ Patient satisfaction scores were higher in the SPB group

\section{Pain intensity}

Different investigators recorded this outcome on different scales and at different intervals. We normalized all NRS to a zero to 10 range (see Table 9). The majority of authors reported pain intensity at $1,6,24$ and $72 \mathrm{~h}$ after surgery.

As shown in Fig. 5, the average NRS scores were $3.4 \pm$ 0.5 at $1 \mathrm{~h}, 3.0$ at $6 \mathrm{~h}, 3.65 \pm 1.15$ at $24 \mathrm{~h}$ and $3.05 \pm 0.25$ at $72 \mathrm{~h}$ in the intervention group; in the placebo group NRS scores were recorded of $5.25 \pm 0.05$ at $1 \mathrm{~h}, 3.0$ at 6 $h, 3.65 \pm 1.15$ at $24 \mathrm{~h}$ and $4.2 \pm 0.5$ at $72 \mathrm{~h}$.

\section{Opioid consumption}

In Gardiner et al [24], 6 patients in the placebo group required supplementary opioid use. No patient received opioids in the intervention group.

After 24 h in Schuitemaker et al [26], the overall mean amounts of morphine consumed in the intervention and placebo groups were $0.2 \pm 0.8 \mathrm{mg}$ and $0.6 \pm 1.2 \mathrm{mg}$, respectively, with the difference being not statistically significant $(p=0.29)$.

No data on opioid consumption was available in Picard et al [25].

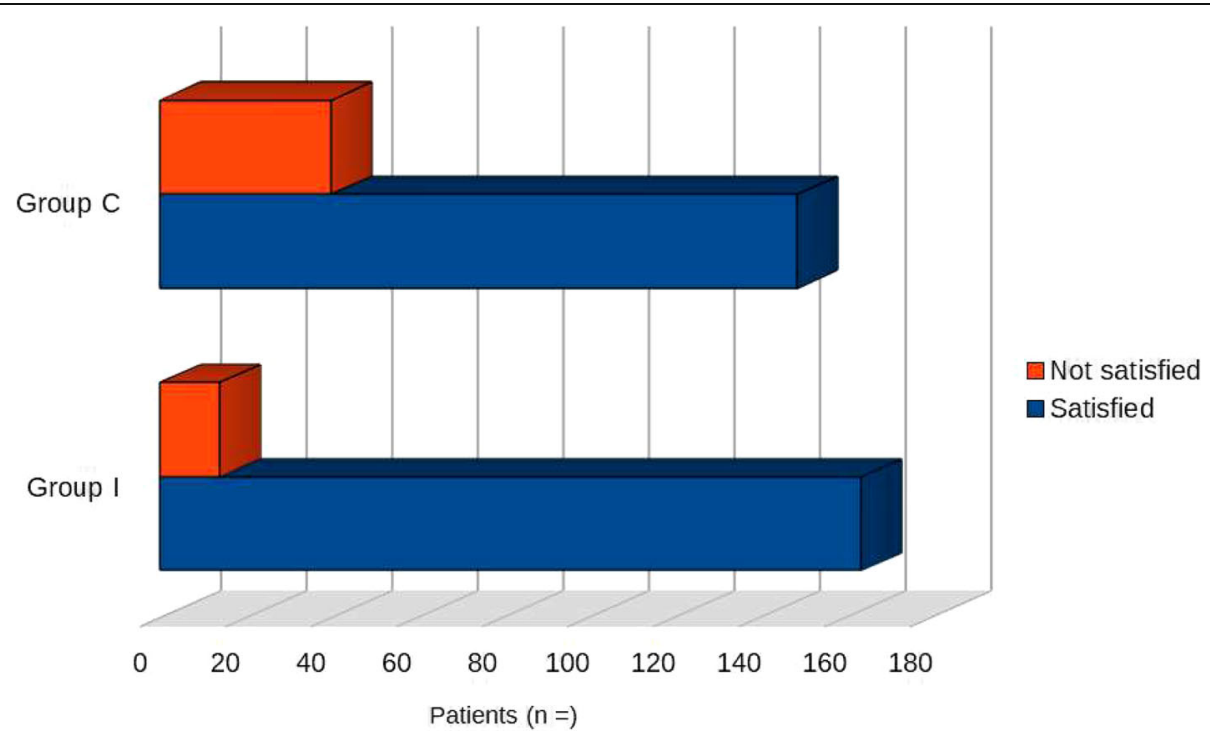

Fig. 4 Patient satisfaction after lumpectomy/mastectomy 
Table 8 Personal and clinical characteristics of patients undergoing breast augmentation

\begin{tabular}{|c|c|c|c|c|c|c|c|c|c|c|}
\hline & \multicolumn{2}{|c|}{ Patients $(n=)$} & \multicolumn{2}{|l|}{ Age (years) } & \multicolumn{2}{|c|}{ Weight (kg) } & \multicolumn{2}{|c|}{ Height $(\mathrm{cm})$} & \multicolumn{2}{|l|}{ BMI $\left(\mathrm{kg} / \mathrm{m}^{2}\right)$} \\
\hline & Group I & Group C & Group I & Group C & Group I & Group C & Group I & Group C & Group I & Group C \\
\hline Gardiner et al., 2012 [24] & 20 & 20 & 33.4 & 34.9 & - & - & - & - & 20.5 & 20.1 \\
\hline Picard et al., 2017 [25] & 29 & 43 & 32.4 & 34.6 & - & - & - & - & - & - \\
\hline $\begin{array}{l}\text { Schuitemaker et al., } \\
2019[26]\end{array}$ & 15 & 15 & 33.0 & 33.0 & 52.0 & 54.0 & 163.0 & 163.0 & 20.0 & 20.0 \\
\hline TOTAL & 64 & 78 & $32.93 \pm 0.41$ & $34.16 \pm 0.83$ & 52.0 & 54.0 & 163.0 & 163.0 & $20.25 \pm 0.25$ & $20.05 \pm 20.04$ \\
\hline
\end{tabular}

\section{Adverse events (AEs)}

A total of 19 (9 vs 10) AEs were recorded.

Gardiner et al [24] reported 12 episodes of nausea and vomiting ( 5 vs $7, p=0.36$ ). In this study, hypotension occurred 3 times among patients in the intervention group and once in the placebo group. A single case of bradycardia occurred in both groups.

No differences were observed between groups concerning the appearance of AEs in Schuitemaker et al [26].

No adverse effects were reported in Picard et al 2017.

\section{Patient satisfaction}

Only Schuitemaker et al [26] reported data about patient satisfaction: after $24 \mathrm{~h}, 80 \%$ vs $53 \%$ of patients in intervention and placebo groups were satisfied.

\section{Breast reduction}

Seventy-nine patients underwent breast reduction. The mean age was $38.28 \pm 2.71$ years vs $38.78 \pm 3.21$ years (see Table 10).

All patients underwent breast reduction surgery.

Tumescent anesthesia was performed in Christie et al 2017; in Valente et al 2014 [28], patients received local infiltration. All patients in these studies underwent general anesthesia.

\section{Pain intensity}

Different investigators recorded this outcome on different scales and at different intervals. We normalized all NRS to a zero to 10 range (see Table 11). The authors reported pain intensity at $24 \mathrm{~h}$ after surgery.
The average NRS score was $2.55 \pm 1.72$ in the intervention group; in the placebo group a NRS score was recorded of $2.85 \pm 1.14(p=0.89)$.

\section{Opioid consumption}

After $24 \mathrm{~h}$ in Christie et al 2017, the overall mean amounts of morphine consumed in the intervention and placebo groups were $0.58 \mathrm{mg}$ and $0.64 \mathrm{mg}$, respectively, with the difference being not statistically significant $(p=0.71)$.

No data on opioid consumption is available in Valente et al 2014 [28].

\section{Adverse events (AEs)}

There was no significant difference in occurrence of nausea or vomiting in the first $24 \mathrm{~h}$ between the two groups in Christie et al 2017. No adverse effects were reported in Valente et al 2014 [28].

\section{Patient satisfaction}

The level of satisfaction was not reported in these studies.

\section{Discussion}

Our systematic review is the first to demonstrate the analgesic benefits of locoregional anesthesia following breast surgery, and to demonstrate the clinical utility of these techniques. For perioperative and postoperative analgesia, locoregional anaesthesia can be used as a standalone anaesthetic technique, or in association with sedation or general anaesthesia. In almost all the studies of this review, locoregional anesthesia was performed to reduce pain as an "adjuvant technique".

Table 9 NRS at 1, 6, 24 and $72 \mathrm{~h}$ after breast augmentation

\begin{tabular}{|c|c|c|c|c|c|c|c|c|}
\hline & \multicolumn{2}{|l|}{ Up to $1 \mathrm{~h}$} & \multicolumn{2}{|c|}{ Up to $6 \mathrm{~h}$} & \multicolumn{2}{|l|}{ Up to $24 \mathrm{~h}$} & \multicolumn{2}{|l|}{ Up to $72 \mathrm{~h}$} \\
\hline & Group I & Group C & Group I & Group C & Group I & Group C & Group I & Group C \\
\hline Gardiner et al., 2012 [24] & 3.9 & 5.2 & - & - & - & - & 3.3 & 4.7 \\
\hline Picard et al., 2017 [25] & - & - & - & - & 4.8 & 5.1 & 2.8 & 3.7 \\
\hline $\begin{array}{l}\text { Schuitemaker et al., } \\
2019[26]\end{array}$ & 2.9 & 5.3 & 3.0 & 3.0 & 2.5 & 3.0 & - & - \\
\hline TOTAL & $3.4 \pm 0.5$ & $5.25 \pm 0.05$ & 3.0 & 3.0 & $3.65 \pm 1.15$ & $4.05 \pm 1.05$ & $3.05 \pm 0.25$ & $4.2 \pm 0.5$ \\
\hline$p$ & 0.06 & & - & & 0.82 & & 0.17 & \\
\hline
\end{tabular}




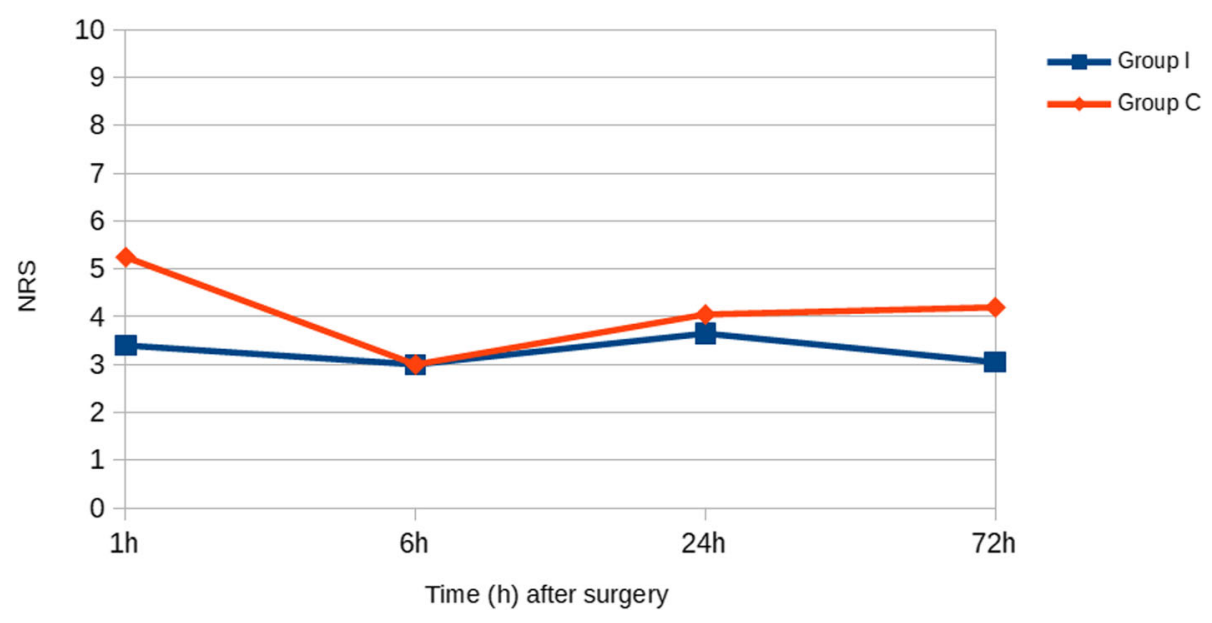

Fig. $5 \mathrm{NRS}$ at 1, 6, 24 and $72 \mathrm{~h}$ after breast augmentation

\section{Lumpectomy/mastectomy \\ Pain intensity}

Pain intensity on a numeric rating scale (NRS) was lower in the intervention group than in the placebo group at 1 , $6,12,24$ and $48 \mathrm{~h}$ after surgery. The difference of NRS at $1 \mathrm{~h}$ between the groups was statistically significant $(p=0.02)$; no statically significant difference was reported at other intervals.

In the first hour after surgery, all patients in the intervention group reported NRS lower than 4; instead in the placebo group, two studies (Ferreira Laso et al., 2014; Wang et al., 2018) experienced, respectively, a mean pain intensity of 6.7 and 4.3. In the placebo group we also found NRS higher than 4 in Wang et al 2019 after $6 \mathrm{~h}$ and in Ferreira Laso et al 2014 after $24 \mathrm{~h}$.

We considered NRS lower than 4 as an optimal cut-off point between mild and moderate pain. This cut-off was identified as the tolerable pain threshold [29].

\section{Opioid consumption}

Postoperative use of opioids was lower in the interventional group both upon discharge from the PACU and after $48 \mathrm{~h}$, although there was no statistically significant difference. The difference in opioid use was statistically significant in three studies (Ferreira Laso et al., 2014; Ilfeld et al., 2014; Versyck et al. 2017) at the time of discharge from the PACU. Eight studies (Campbell et al., 2014 [10]; Ferreira Laso et al., 2014; Gürkan et al., 2018;
Mohamed et al., 2013; Neethu et al., 2018; Versyck et al. 2017; Wang et al., 2018; Yao et al., 2019) reached statistical significance after $48 \mathrm{~h}$ from surgery.

\section{Safety}

No statistically significant difference was noticed ( $p=$ 0.74) between interventional and placebo groups. Among the intervention group a total of 163 AEs was reported. Nausea, vomiting or PONV were the most common (59\%), followed by bruising (14\%), haematoma/ bleeding (6\%), seroma (6\%), pruritus $(4 \%)$ and haemodynamic alterations (4\%), such as hypotension, hypertension or bradycardia.

\section{Patient satisfaction}

Patient satisfaction was high, with minimum $92 \%$ of satisfaction among patients treated with locoregional anesthesia. The satisfaction rate was also high in the placebo group (79\%).

\section{Breast augmentation}

Pain intensity on a numeric rating scale (NRS) was lower in the intervention group than in the placebo group at 1 , 6,24 and $72 \mathrm{~h}$ after surgery. No statically significant difference was reported at these intervals.

Postoperative use of opioids was lower in the interventional group after $24 \mathrm{~h}$, although there was no statistically significant difference.

Table 10 Personal and clinical characteristics of patients undergoing breast reduction

\begin{tabular}{|c|c|c|c|c|c|c|c|c|c|c|}
\hline & \multicolumn{2}{|c|}{ Patients, $n$} & \multicolumn{2}{|l|}{ Age, years } & \multicolumn{2}{|c|}{ Weight, kg } & \multicolumn{2}{|c|}{ Height, cm } & \multicolumn{2}{|c|}{$B M I, k g / m^{2}$} \\
\hline & Group I & Group C & Group I & Group C & Group I & Group C & Group I & Group C & Group I & Group C \\
\hline Christie et al., 2017 [27] & 20 & 20 & 41.0 & 42.0 & - & - & - & - & 33.0 & 31.0 \\
\hline Valente et al., 2014 [28] & 18 & 20 & 35.57 & 35.57 & - & - & - & - & - & - \\
\hline TOTAL & 38 & 40 & $38.28 \pm 2.71$ & $38.78 \pm 3.21$ & - & - & - & - & 33.0 & 31.0 \\
\hline
\end{tabular}


Table 11 NRS at $24 \mathrm{~h}$ after breast reduction

\begin{tabular}{lll}
\hline & Up to $\mathbf{2 4} \mathbf{~}$ \\
\cline { 2 - 3 } & Group I & Group C \\
\hline Christie et al., 2017 [27] & 4.28 & 4.00 \\
Valente et al., 2014 [28] & 0.83 & 1.71 \\
TOTAL & $\mathbf{2 . 5 5} \pm \mathbf{1 . 7 2}$ & $\mathbf{2 . 8 5} \pm \mathbf{1 . 1 4}$ \\
$\boldsymbol{p}$ & $\mathbf{0 . 8 9}$ & \\
\hline
\end{tabular}

Concerning safety, no difference was noticed between interventional and placebo groups.

\section{Breast reduction}

Pain intensity on a numeric rating scale (NRS) was lower in the intervention group than in the placebo group after $24 \mathrm{~h}$. No statically significant difference was reported.

Postoperative use of opioids was lower in the interventional group after $24 \mathrm{~h}$, although there was no statistically significant difference.

No difference was noticed between interventional and placebo groups about AEs incidence.

General anesthesia is the conventional, most frequently used anesthetic technique. Various locoregional anesthetic techniques are also used for breast surgeries. These include local wound infiltration [30], tumescent anesthesia [31], regional anesthetic techniques, such as pectoral nerve (Pecs) blocks type 1 and 2 [32, 33], serratus plane block (SPB) [34], and parasternal block (PSB) [35], pain pump $[36,37]$, and intravenous regional block [7, 38].

We considered continuous IV infusion of lidocaine for our review. Various are the reasons that led us to consider this technique. Local anesthetics exert their pharmacological action through the block of sodium channels in neural tissues, thereby interrupting neuronal transmission. This action is best demonstrated when the drug comes directly in contact of neural tissues. However, the systemic effects of lidocaine are also probably or at least partially, related to this mechanism [39]. The IV lidocaine shares many of the effects of local anesthetics when used in regional anesthesia techniques. It can lead to better postoperative analgesia, reduced opioid consumption and improved intestinal motility [40]. In addition to the analgesic action, local anesthetics have anti-inflammatory action, justifying also the use of intravenous lidocaine to modulate the inflammatory response resulting from postoperative pain [41].

Locoregional anesthesia provides effective anesthesia and analgesia in the perioperative setting. The beneficial analgesic effect of the regional block is well known, and also confirmed in our analysis. After mastectomy, the use of locoregional anesthesia techniques seems to reduce pain especially in the first hour after the end of the surgery.

Other potentially beneficial effects of locoregional anaesthesia and analgesia on other perioperative outcomes include decreased need for opioids for controlling postoperative pain, decreased postoperative nausea and vomiting, fewer complications and increased patient satisfaction. In our review, there was no statistically significant difference between the analysed anesthesia techniques.

The effective management and relief of postoperative pain plays a vital role in overall surgical outcome. Untreated pain has been linked to prolonged hospital stays, deep venous thrombosis, pulmonary embolism, pneumonia, bowel dysmotility, insomnia, and impaired wound healing [42]. Reduced occurrence of nausea and vomiting is related to better analgesia and opioids/inhalational anaesthetics sparing effect by regional blocks [43, 44]. All this improves post-operative recovery and shortens hospitalization stay.

\section{Limitations}

Our review has several limitations. First, some outcomes were characterized by high levels of heterogeneity. Reasons for this may be attributable to subtle variations in surgical technique and differences in anesthetic and analgesic regimens. Second, for 3 studies included in this review (Campbell et al 2014 [10], Lanier et al 2018 and Picard et al [25]), it is not possible to assess whether only regional anesthesia for breast surgery was performed. The impact of locoregional anesthesia on nociception as a "pure" or "adjuvant" technique is different, notably because of the different dosage of local anesthetics. We decided not to exclude these studies and to accept the possible bias during the analysis. Third, many of the included studies had small sample sizes, which decreases their effect and limits external validity. Fourth, another major limitation of this review was the large and unexplained statistical heterogeneity between the studies. Finally, we included two studies (Couceiro et al [11]; Terkawi et al 2014) analysing the use of i.v. lidocaine. Systemic lidocaine is not "really" a locoregional anesthesia technique, nevertheless we decided to include it in our review accepting the possible bias arising from systemic effects of this local anesthetic.

All these limitations reduced the quality of the evidence for most of the outcomes.

\section{Conclusion}

In this systematic review we found evidence for an effect of locoregional anesthesia on the pain due to breast surgery as one the major predefined outcomes. The difference of NRS at $1 \mathrm{~h}$ between the groups was statistically significant among patients who underwent lumpectomy/mastectomy; no statically significant difference was reported at other intervals.

The postoperative opioids consumption, the incidence of PONV and other AEs, and the patient satisfaction were not different among patients who underwent locoregional anesthesia or conventional analgesia. 


\section{Abbreviations}

AEs: Adverse Events; ALND: Axillary Lymph Node Dissection; BMI: Body Mass Index; DVT: Deep Vein Thrombosis; ESP: Erector Spinae Plane; IV: Intravenous; NRS: Numeric Rating Scale; PACU: Post-Anesthesia Care Unit; Pecs: Pectoralis nerve; PONV: Postoperative Nausea and Vomiting; PTE: Pulmonary Thromboembolism; PVB: Paravertebral Block; SLNB: Sentinel Lymph Node Biopsy; SPB: Serratus Plane Block

\section{Acknowledgements}

Not applicable.

\section{Disclosures}

Pasquale Sansone, Luca G. Giaccari, Mario Faenza, Pasquale Di Costanzo, Sara Izzo, Caterina Aurilio, Francesco Coppolino, Maria Beatrice Passavanti, Vincenzo Pota and Maria Caterina Pace have nothing to disclose.

\section{Authors' contributions}

PS helped design the study, conduct the study, analyse the data, and write the manuscript; LGG helped design the study, conduct the study, analyse the data, and write the manuscript; MF helped design the study and analyse the data; PDC helped design the study and analyse the data; SI helped design the study and analyse the data; CA helped design the study and analyse the data; FC helped design the study and analyse the data; MBP helped design the study and analyse the data; VP helped design the study and analyse the data; MCP helped design the study, conduct the study and analyse the data. All authors have read and approved the manuscript.

\section{Funding}

No funding or sponsorship was received for this study or publication of this article.

\section{Availability of data and materials}

Dataset derived from public resources and are stored in our computer system at the anesthesia institute of the University Hospital "Luigi Vanvitelli" (Naples - Italy). Datasets are available from the corresponding author on reasonable request.

\section{Ethics approval and consent to participate}

This article is based on previously conducted studies and does not contain any studies with human participants or animals performed by any of the authors.

\section{Consent for publication}

Not applicable.

\section{Competing interests}

The authors declare that they have no competing interests.

\section{Author details}

'Department of Woman, Child and General and Specialized Surgery, University of Campania "Luigi Vanvitelli", Piazza Luigi Miraglia, 2, Naples, Italy. ${ }^{2}$ Multidisciplinary Department of Medical Surgical and Dental Sciences Plastic Surgery Unit, University of Campania "Luigi Vanvitelli", Naples, Italy.

Received: 27 May 2020 Accepted: 16 November 2020 Published online: 23 November 2020

\section{References}

1. Siegel RL, Miller KD, Jemal A. Cancer statistics, 2020. CA A Cancer J Clin. 2000;70:7-30.

2. American Society of Plastic Surgeons. Plastic Surgery Statistics Report https://www.plasticsurgery.org/documents/News/Statistics/2018/plasticsurgery-statistics-full-report-2018.pdf. Accessed 13 Apr 2020.

3. Broyles JM, Tuffaha SH, Williams EH, Glickman L, George TA, Lee DA. Pain after breast surgery: etiology, diagnosis, and definitive management. Microsurgery. 2016;36(7):535-8.

4. Pace MC, Mazzariello L, Passavanti MB, Sansone P, Barbarisi M, Aurilio C. Neurobiology of pain. J Cell Physiol. 2006;209(1):8-12.

5. Chou R, Gordon DB, de Leon-Casasola OA, et al. Management of Postoperative Pain: a clinical practice guideline from the American pain society, the American Society of Regional Anesthesia and Pain Medicine, and the American Society of Anesthesiologists' committee on regional anesthesia, executive committee, and administrative council. J Pain. 2016; 17(2):131-57.

6. Wick EC, Grant MC, Wu CL. Postoperative multimodal analgesia pain management with nonopioid analgesics and techniques: a review. JAMA Surg. 2017;152(7):691-7.

7. Dunn LK, Durieux ME. Perioperative Use of Intravenous Lidocaine. Anesthesiology. 2017;126(4):729-37.

8. Moher D, Liberati A, Tetzlaff J, Altman DG, The PRISMA Group. Preferred Reporting Items for Systematic Reviews and Meta-Analyses: The PRISMA Statement. BMJ. 2009;339:b2535.

9. Equivalent Opioid Calculator (EOC). Available at http://clincalc.com/Opioids/. Accessed 6 Apr 2020.

10. Campbell I, Cavanagh S, Creighton J, et al. Local anaesthetic in breast surgery. ANZ J Surg. 2015;85:353-7.

11. Couceiro TC, Lima LC, Burle LM, Valença MM. Intravenous lidocaine for postmastectomy pain treatment: randomized, blind, placebo controlled clinical trial. Braz J Anesthesiol. 2015;65(3):207-12.

12. Cros J, Sengès $P$, Kaprelian $S$, et al. Pectoral I block does not improve postoperative analgesia after breast Cancer surgery: a randomized, doubleblind, dual-centered controlled trial. Reg Anesth Pain Med. 2018:43:596-604.

13. Ferreira Laso L, Lopez-Picado A, Lamata L, et al. Postoperative Analgesia by Infusion of Local Anesthetic into the Surgical Wound after Modified Radical Mastectomy. Plast Reconstr Surg. 2014;134(6):p862e-70e.

14. Gürkan Y, Aksu C, Kuș A, Yörükoğlu UH, Kılıç CT. Ultrasound guided erector spinae plane block reduces postoperative opioid consumption following breast surgery: a randomized controlled study. J Clin Anesth. 2018;50:65-8.

15. Iffeld BM, Madison SJ, Suresh PJ, et al. Treatment of postmastectomy pain with ambulatory continuous paravertebral nerve blocks: a randomized, triple-masked, placebo-controlled study. Reg Anesth Pain Med. 2014;39(2):89-96.

16. Lanier ST, Lewis KC, Kendall MC, et al. Intraoperative nerve blocks fail to improve quality of recovery after tissue expander breast reconstruction: a prospective, double-blinded, randomized, Placebo-Controlled Clinical Trial. Plast Reconstr Surg. 2018;141(3):590-7.

17. Mohamed SA, Abdel-Ghaffar HS. Effect of the addition of clonidine to locally administered bupivacaine on acute and chronic postmastectomy pain. J Clin Anesth. 2013;25(1):20-7.

18. Neethu M, Pandey RK, Sharma A, et al. Pectoral nerve blocks to improve analgesia after breast cancer surgery: a prospective, randomized and controlled trial. J Clin Anesth. 2018;45:12-7.

19. Terkawi AS, Durieux ME, Gottschalk A, et al. Effect of intravenous Lidocaine on postoperative recovery of patients undergoing mastectomy: a doubleblind, placebo-controlled randomized trial. Reg Anesth Pain Med. 2014;39: 472-7.

20. Versyck B, van Geffen CJ, Van Houwe P. Prospective double blind randomized placebo-controlled clinical trial of the pectoral nerves (Pecs) block type II. J Clin Anesth. 2017;40:46-50

21. Wang $K$, Zhang $X$, Zhang $T$, et al. The efficacy of ultrasound-guided type I pectoral nerve blocks in perioperative pain Management for Immediate Reconstruction after Modified Radical Mastectomy: a prospective, Randomized Study. Clin J Pain. 2018;34(3):231-6.

22. Wang $W$, Song $W$, Yang $C$, et al. Ultrasound-guided pectoral nerve block I and Serratus-intercostal plane block alleviate postoperative pain in patients undergoing modified radical mastectomy. Pain Physician. 2019;22(4):E315-23.

23. Yao Y, Li J, Hu H, Xu T, Chen Y. Ultrasound-guided serratus plane block enhances pain relief and quality of recovery after breast cancer surgery. Eur J Anaesthesiol. 2019;36(6):436-41.

24. Gardiner S, Rudkin G, Cooter R, Field J, Bond M. Paravertebral blockade for day-case breast augmentation. Anesth Analg. 2012;115(5):1053-9.

25. Picard F, Niddam J, De Runz A, Chaouat M, Mimoun M, Boccara D. Intraoperative instillation of ropivacaine during the placement of submuscular cosmetic breast implants: is there a clinical benefit? Ann Chir Plast Esthet. 2018:63(1):20-4.

26. Schuitemaker RJB, Sala-Blanch AP, Sánchez Cohen LA, López-Pantaleon JT, Mayoral R, Cubero M. Analgesic efficacy of modified pectoral block plus serratus plane block in breast augmentation surgery: a randomised, controlled, triple-blind clinical trial. Rev Esp Anestesiol Reanim. 2019;66(2):62-71.

27. Christie BM, Kapur S, Kempton SJ, Hanson SE, Ma Y, Rao VK. A Prospective Randomized Trial Comparing the Effects of Lidocaine in Breast Reduction Surgery. Plast Reconstr Surg. 2017;139(5):1074e-9e. 
28. Valente DS. Preemptive analgesia with bupivacaine in reduction Mammaplasty. Plast Reconstr Surg. 2014;134(4):581-6.

29. Gerbershagen HJ, Rothaug J, Kalkman CJ, Meissner W. Determination of moderate-to-severe postoperative pain on the numeric rating scale: a cutoff point analysis applying four different methods. Br J Anaesth. 2011;107(4): 619-26.

30. Mustoe TA, Buck DW, Lalonde DH. The safe management of anesthesia, sedation and pain in plastic surgery. Plast Reconstr Surg J. 2010;126(4): 165e-76e.

31. Khater A, Mazy A, Gad M, Taha Abd Eldayem O, Hegazy M. Tumescent mastectomy: the current indications and operative tips and tricks. Breast Cancer Targets Ther. 2017;9:237-43.

32. Blanco R. The 'pecs block': a novel technique for providing analgesia after breast surgery. Anaesthesia. 2011;66:840-52.

33. Blanco R, Fajardo M, Parras MT. Ultrasound description of Pecs II (modified Pecs I): a novel approach to breast surgery. Rev Esp Anestesiol Reanim. 2012;59:470-5

34. Blanco R, Parras T, McDonnell JG, Prats-Galino A. Serratus plane block: a novel ultrasound-guided thoracic wall nerve block. Anaesthesia. 2013;68(11): 1107-13.

35. Fusco P, Scimia P, Petrucci E, DI Carlo S, Marinangeli F. The ultrasoundguided parasternal block: a novel approach for anesthesia and analgesia in breast cancer surgery. Minerva Anestesiol. 2017;83(2):221-2.

36. Chandran GJ, Lalonde DH. A review of pain pumps in plastic surgery. Can J Plast Surg. 2010;18(1):15-8.

37. Monahan AM, Iffeld BM. Continuous peripheral nerve blocks. In: Hadzic A, editor. Hadzic's textbook of regional anesthesia and acute pain management. 2th ed. United States: McGraw Hill Companies; 2017. p. 1180-6.

38. Chang YC, Liu CL, Liu TP, Yang PS, Chen MJ, Cheng SP. Effect of perioperative intravenous lidocaine infusion on acute and chronic pain after breast surgery: a meta-analysis of randomized controlled trials. Pain Pract. 2017;17(3):336-43.

39. de Oliveira CM, Issy AM, Sakata RK. Intraoperative intravenous lidocaine. Rev Bras Anestesiol. 2010;60(3):325-33.

40. Kranke $\mathrm{P}$, Jokinen J, Pace $\mathrm{NL}$, et al. Continuous intravenous perioperative lidocaine infusion for postoperative pain and recovery. Cochrane Database Syst Rev. 2015;7:CD009642.

41. Clarke C, McConachie I, Banner R. Lidocaine infusion as a rescue analgesic in the perioperative setting. Pain Res Manag. 2008;13:421-3.

42. Carr DB, Goudas L. Acute pain. Lancet. 1999;353:2051

43. Cali Cassi L, Biffoli F, Francesconi D, Petrella G, Buonomo O. Anesthesia and analgesia in breast surgery: the benefits of peripheral nerve block. Eur Rev Med Pharmacol Sci. 2017;21:1341-5.

44. Becker DE. Nausea, vomiting, and hiccups: a review of mechanisms and treatment. Anesth Prog. 2010;57:150-6.

\section{Publisher's Note}

Springer Nature remains neutral with regard to jurisdictional claims in published maps and institutional affiliations.

Ready to submit your research? Choose BMC and benefit from:

- fast, convenient online submission

- thorough peer review by experienced researchers in your field

- rapid publication on acceptance

- support for research data, including large and complex data types

- gold Open Access which fosters wider collaboration and increased citations

- maximum visibility for your research: over $100 \mathrm{M}$ website views per year

At $\mathrm{BMC}$, research is always in progress.

Learn more biomedcentral.com/submissions 